iassns-hep-92-92

\title{
Skyrmions and Semilocal Strings in Cosmology
}

\author{
Katherine Benson $^{\dagger}$ and Martin Bucher ${ }^{\ddagger}$ \\ School of Natural Science, Institute for Advanced Study \\ Olden Lane, Princeton, New Jersey 08540
}

\begin{abstract}
It has been pointed out that cosmic string solutions can exist in gauge field theories with broken symmetry even when $\pi_{1}(G / H)$ is trivial. The stability of such semilocal defects is not guaranteed by topology and depends on dynamical considerations. In the literature it has been tacitly assumed that if stable, such strings would form in the Early Universe in a manner analogous to the formation of a network of more robust topologically-stable strings. In this paper we find that except for unnaturally small values of the correlation length, a network of semilocal strings does not form. Instead, delocalized skyrmionic string configurations, which expand with the Hubble flow, dominate.
\end{abstract}

April 1993

$\dagger$ Supported in part by NSF grant PHY91-6210

$\ddagger$ Supported in part by the US Department of Energy grant DE-FG02-90ER40542 


\section{Introduction}

When there is a pattern of symmetry breaking $G \rightarrow H$ through the formation of a condensate, there is a manifold of degenerate vacua that is homeomorphic to the coset space $G / H$. When the homotopy groups $\pi_{k}(G / H)$ are nontrivial, topologically-stable defects arise in the theory. ${ }^{[1,2]}$ In $3+1$ dimensions, domain walls result for nontrivial $\pi_{0}(G / H)$, cosmic strings for nontrivial $\pi_{1}(G / H)$, monopoles for nontrivial $\pi_{2}(G / H)$, and textures for nontrivial $\pi_{3}(G / H)$. The physics of such defects in quantum field theory has been studied in much detail. Topological defects form the basis of several proposed scenarios for structure formation in the Early Universe. ${ }^{[3]}$

Recently there has been much discussion of embedded defects, especially embedded strings, which may exist even when $\pi_{1}(G / H)$ is trivial ${ }^{[6-19]}$. Like their conventional counterparts, embedded strings, with a Higgs field winding about a line defect carrying magnetic flux, satisfy the classical field equations. However, because they arise in theories with trivial $\pi_{1}(G / H)$, the stability of these embedded solutions does not follow from topological considerations, but rather depends on dynamical considerations. Despite their more fragile basis, nontopological strings are stable against small perturbations for a natural range of parameters.

The simplest model with embedded strings is an extension of the $U(1)$ abelian Higgs model with the familiar topologically-stable Abrikosov-Nielsen-Oleson strings $^{[4,5]}$. In this extended model, considered by Vachaspati and Achúcarro ${ }^{[6]}$, the complex Higgs singlet is replaced by a complex Higgs doublet, so that in addition to the gauged $U(1)$ local symmetry, there is also an $S U(2)$ global symmetry, and the vacuum manifold has the topology of $S^{3}$. The action of $U(1)$ foliates $S^{3}$ into gauge orbits, each with the structure of $S^{1}$. For the embedded strings - called "semilocal strings" by Vachaspati and Achúcarro - the Higgs field is restricted to one of these gauge orbits. The string is not topologically stable because the gauge field can unwind by slipping off the gauge orbit. However, such a perturbation need not lower the energy

because the scalar gradient energy is increased by the perturbations. Hindmarsh ${ }^{[9]}$ examined the issue of stability in this model and found that stability is determined 
by the dimensionless parameter $\beta=m_{H}^{2} / m_{V}^{2}=\lambda / g^{2}$. For $\beta<1$ the solution is stable against small perturbations; for $\beta>1$ the solution is unstable.

Since semilocal strings are not necessarily stable, the question arises: Into what can they decay? In the model described above, there is a competing family of configurations, considered by both Hindmarsh and Preskill ${ }^{[9,19,18]}$, dubbed "skyrmions" by Preskill. These "skyrmions" are essentially global textures in two spatial dimensions - or alternatively, fat delocalized strings. At very large radii, the Higgs field winds around a gauge orbit and is compensated by the gauge field so that the covariant derivative vanishes, just as for the localized semilocal strings. However, inside the core of the skyrmion the Higgs field does not vanish as in the AbrikosovNielsen-Oleson solution, but rather remains on (or very close to) the vacuum manifold. Said another way, the twist around a gauge orbit at spatial infinity unwinds in the core of the skyrmion while the Higgs field essentially remains on the vacuum manifold. The existence of such skyrmionic configurations follows directly from the triviality of $\pi_{1}(G / H)$. Because the Higgs field need not leave the vacuum manifold to unwind the twist at infinity, skyrmions can be made arbitrarily large. For very large skyrmions, the tension is dominated by scalar gradient energy that cannot be compensated by the gauge field. The tension of a large skyrmion (that is, $R \gg v^{-1}$ ) is of order $v^{2}$, where $v$ is the symmetry breaking scale. It is a dynamical question whether this tension is greater than or less than the tension of the Abrikosov-Nielsen-Oleson string, which also is of order $v^{2}$.

The existence of competing low-energy, delocalized skyrmion configurations complicates the analysis of the role of semilocal strings in cosmology. Because the frustration generated by quenching in a cosmological phase transition can be resolved either through the formation of localized semilocal strings or through the formation of delocalized skyrmionic strings, the usual analysis of string formation and evolution breaks down, and an understanding of skyrmion dynamics is crucial to determining the role of semilocal strings in cosmology.

Both Hindmarsh and Preskill have considered the dynamics of skyrmions ${ }^{[18,9,19]}$. 
Preskill considered skyrmions in the $C P^{n}$ limit, in which the Higgs field is constrained to the vacuum manifold, and suggested that large skyrmions always tend to expand, because the magnetic energy, which breaks the approximate scale-invariance of the string tension, gives smaller skyrmions a larger energy. Hindmarsh examined the special case $\beta=1$ and demonstrated the existence of a one-parameter family of static solutions to the equations of motion that continuously interpolates between the embedded vortex solutions and skyrmion configurations of arbitrarily large size. Hindmarsh also considered how the energy of these exact solutions for $\beta=1$ varies as $\beta$ is perturbed away from one and concluded that for $\beta>1$ skyrmions want to expand, and for $\beta<1$ skyrmions want to contract.

It is interesting to consider what happens when the global symmetry in a model with semilocal strings is gauged. For the $S U(2)_{\text {global }} \times U(1)_{\text {local }}$ model considered by Vachaspati and Achúcarro, the result is essentially the standard electroweak model. The physics of embedded strings in the Weinberg-Salam electroweak model was first investigated by Y. Nambu in $1977^{[7]}$, and more recently by Vachaspati and collaborators $^{[8,11,12]}$, who explicitly analyzed the issue of stability. The stability of "electroweak" strings depends on $\beta=\left(\lambda / g^{\prime 2}\right)$ and the ratio $\left(g / g^{\prime}\right)$. The region of stability in the $\beta$ - $\left(g / g^{\prime}\right)$ plane is a strip where $\beta<1$ and $g / g^{\prime}$ is small. In other words, the stability is not destroyed when $S U(2)$ is gauged very weakly compared to $U(1)$.

These embedded electroweak string are not "semilocal" because every direction on the vacuum manifold can be compensated by the gauge field $A_{\mu}$. (We shall call such strings "completely-gauged" nontopological strings, as opposed to "semilocal" nontopological strings.) The crucial difference is that for completely-gauged nontopological strings there are no large competing skyrmions (because the scalar gradients can be completely compensated by the gauge field), and the embedded string can break instead of transforming into a skyrmion, which in essence is just a fattened string. The ability of completely-gauged nontopological strings to break makes them even more fragile than their semilocal counterparts - a fact whose cosmological consequences will be discussed more. 
In this paper, we further investigate the dynamics of delocalized "skyrmionic" field configurations. A heuristic discussion of skyrmion dynamics is first presented based on an order-of-magnitude variational calculation. Then a more careful attempt to define skyrmions for $\beta \neq 1$ is presented. The "size" of a skyrmion is defined in terms of its magnetic energy, and the lowest energy configuration of a given size is found. Then the time evolution of the resulting one-parameter family of configurations is examined. The effect of the expansion of the universe is taken into account and found to be significant. We also discuss the role of localized embedded strings and delocalized skyrmionic strings in cosmology. In particular, we discuss the question of whether a network of localized "semilocal" strings would be formed in a cosmological phase transition, emphasizing how the standard analysis for topologically stable strings, which inexorably leads to a network described by the "scaling" solution, breaks down. We conclude that the prospects of forming a network of semilocal strings are rather bleak.

The organization of the paper is the following. Section 2 presents some background material on embedded string and skyrmion configurations. Section 3 deals with skyrmion dynamics. Section 4 discusses the physics of completely-gauged nontopological strings. Section 5 discusses cosmological implications. Section 6 presents some concluding remarks.

\section{Semilocal Strings and Skyrmions: An Inevitable Competition}

The simplest model with nontopological "semilocal" strings has a complex doublet $\Phi=\left(\begin{array}{c}\phi_{1} \\ \phi_{2}\end{array}\right) .{ }^{[6]}$ This adds to the local $U(1)$ symmetry a global $S U(2)$ symmetry-hence the name "semilocal." The action for this model is

$$
S=\int d^{4} x\left\{\frac{-1}{4 g^{2}} F_{\mu \nu} F^{\mu \nu}+\left[\left(\partial_{\mu}-i A_{\mu}\right) \Phi\right]^{\dagger}\left[\left(\partial^{\mu}-i A^{\mu}\right) \Phi\right]-\frac{\lambda}{2}\left[\Phi^{\dagger} \Phi-v^{2}\right]^{2}\right\}
$$

The vacuum manifold $\mathcal{M}=\left\{\phi \mid \phi^{\dagger} \phi=v^{2}\right\}$ has the topology of $S^{3}$, so that $\pi_{1}(G / H)=\pi_{1}\left(S^{3}\right)$ is trivial. However, an embedded Abrikosov-Nielsen-Olesen solu- 
tion still satisfies the equations of motion, with the form

$$
\begin{aligned}
\Phi(r, \theta) & =v \cdot\left(\begin{array}{c}
f(r) e^{i \theta} \\
0
\end{array}\right), \\
\mathbf{A}(r) & =\hat{\mathbf{e}}_{\theta} \cdot \frac{a(r)}{r}
\end{aligned}
$$

where $f(0)=a(0)=0$ and $f(r), a(r) \rightarrow 1$ as $r \rightarrow \infty$. The string core has a characteristic size $r_{v o r} \sim v^{-1}$, inside which $f(r)$ and $g(r)$ deviate significantly from one. For $r \gg r_{v o r}$ the solution becomes pure vacuum, with $\Phi(x)$ at the minimum of the potential and the covariant gradients and gauge field curvature vanishing. The boundary condition at spatial infinity is best understood by foliating the vacuum manifold $S^{3}$ using the action of the gauged $U(1)$ symmetry on $S^{3}$. Passing through each point $\Phi_{0} \in S^{3}$ is a unique gauge orbit that has the topology of $S^{1}$ and coincides with a great circle of $S^{3}$. It is energetically favorable for $\Phi(x)$ to vary in directions along these gauge orbits rather than in directions orthogonal to them, for the gauge field $A_{\mu}$ can compensate only parallel gradients. This is why a configuration with nontrivial winding number in the gauge orbit is not necessarily unstable to slipping off the gauge orbit.

The triviality of the gauge orbit on the vacuum manifold, however, implies the existence of competing skyrmionic strings. Let us fix the fields at large $R$ so that $\Phi(x)$ lies entirely within a single gauge orbit. Let $\Phi(x)$ have a nontrivial winding number, with $\mathbf{A}(x)$ completely compensating the gradient in $\Phi(x)$ at large radii. The fact that $\pi_{1}(G / H)$ is trivial in the semilocal case indicates that there exist field configurations with this boundary for which $\Phi(x)$ nowhere deviates from the vacuum manifold. For the $S U(1)_{\text {global }} \times U(1)_{\text {local }}$ model, an ansatz for configurations of this sort is

$$
\begin{gathered}
\Phi(r, \theta)=v \cdot\left(\begin{array}{c}
f(r) e^{i \theta} \\
g(r)
\end{array}\right), \\
A(r)=\hat{e}_{\theta} \cdot \frac{a(r)}{r}
\end{gathered}
$$

where $f(0)=a(0)=0, g(0)=1, f(r)^{2}+g(r)^{2}=1$ for all $r$, and as $r \rightarrow \infty$, $f, a \rightarrow 1$ and $g \rightarrow 0$. At large radii, $\Phi(r, \theta)$ lies on a gauge orbit, which can be 
thought of as the equator of a three-sphere. As $r$ is decreased, $\Phi(r, \theta)$ slips off the equator toward one of the poles. When $r=0$, the loop has contracted to a point. The resulting configuration resembles a two-dimensional texture-hence the name "skyrmion." Excluding the magnetic energy and restricting the Higgs field to the vacuum manifold gives an energy for these configurations that is invariant under scale transformations. This is because they have a gradient of order $(v / R)$ spread out over an area $R^{2}$, giving an energy per unit length of order $v^{2}$.

This reveals the crucial difference between field configurations described by the Abrikosov-Nielsen-Olesen ansatz (2.2) and field configurations described by the skyrmion ansatz (2.3) . The size $R$ of a skyrmion configuration is variable and can be made arbitrarily large while keeping the energy bounded from above. This is because $\Phi(x)$ remains on (or very close to) the vacuum manifold. By contrast, AbrikosovNielsen-Olesen configurations (with $g(r)=0$ ) cannot be made arbitrarily large. There is a potential energy cost of order $\lambda v^{4} R^{2}$ associated with a large Abrikosov-NielsenOlesen configuration.

A model that supports semilocal strings therefore has competing solutions - the Abrikosov-Nielsen-Olesen solution with localized magnetic flux and localized deviations from the gauge orbit, and the skyrmion configurations with delocalized flux and delocalized deviation from the gauge orbit. The existence of competing configurations has two important consequences: (1) The stability of the localized solutions is not guaranteed by topological arguments. (2) Even if stable, a localized semilocal string can be unwound with the addition of a finite amount of energy per unit length. When a semilocal string is bombarded with a sufficient amount of energy, it will unwind by becoming an expanding skyrmion solution. The dynamics of this process will be discussed in more detail in section 3.

The stability of semilocal strings to small perturbations depends on the dimensionless parameter $\beta=\left(\lambda / g^{2}\right)=\left(m_{V} / m_{S}\right)^{2}$. $\beta$ is identical to $\kappa$ in superconductivity, which separates Type-I superconductors $(\kappa<1)$ from Type -II superconductors $(\kappa>1)$. For $\beta>1$ the semilocal strings are unstable to small perturbations, but 
for $\beta<1$ they are stable against small perturbations ${ }^{[9]}$. This can be understood heuristically in the following manner. Consider a perturbation of the AbrikosovNielsen-Olesen solution in which the Higgs field in the core is pushed away from $\Phi=0$ toward the vacuum manifold. This perturbation gives a decrease in the potential energy proportional to $\lambda v^{4} r_{v o r}^{2}$ at the expense of an increase in gradient energy proportional to $v^{2}$. Since $r_{v o r} \approx(g v)^{-1}$, the ratio is of order $\lambda / g^{2}$.

\section{Skyrmion Dynamics}

We now discuss the dynamics of skyrmions. In section 2 we noted that excluding magnetic energy and restricting the field $\Phi(x)$ to the vacuum manifold gives a skyrmion energy that is invariant under dilatations. Two effects break this scale invariance, for large skyrmions. First, there is the magnetic energy which scales as $R^{-2}$. Second, there is a correction, also of order $R^{-2}$ but of opposite sign, from the back reaction of the covariant scalar gradient on the potential, which reduces $|\Phi(x)|$ inside the skyrmion. The relative strengths of these effects determine whether initially static skyrmions tend to expand or contract.

Before discussing the general case, we examine the special case $\beta=1$, which lies on the brink of instability for the Abrikosov-Nielsen-Olesen solution. Here the two corrections cancel, yielding a continuous family of static solutions of degenerate energy that interpolate between the smallest semilocal string and the largest skyrmions.

We see this by exploiting a remarkable simplification due to Bogomol'nyi ${ }^{[20]}$. After rescaling fields and spatial coordinates, the static energy functional for the action (2.1) depends only on the parameter $\beta$ :

$$
E=v^{2} \int d^{2} x\left\{\frac{1}{2} B^{2}+\left[\left(\partial_{k}-i A_{k}\right) \Phi\right]^{\dagger}\left[\left(\partial_{k}-i A_{k}\right) \Phi\right]+\frac{\beta}{2}\left[\Phi^{\dagger} \Phi-1\right]^{2}\right\}
$$

For finite energy configurations which wind once around the gauge orbit at spatial 
infinity, Bogomol'nyi rewrites (3.1) as

$$
\begin{aligned}
E / v^{2}=2 \pi+\int d^{2} x & \left\{\frac{1}{2}\left[\left(D_{j} \Phi\right)+i \epsilon_{j k}\left(D_{k} \Phi\right)\right]^{\dagger}\left[\left(D_{j} \Phi\right)+i \epsilon_{j l}\left(D_{l} \Phi\right)\right]\right. \\
+ & \left.\frac{1}{2}\left(\Phi^{\dagger} \Phi-1+B\right)^{2}+\frac{(\beta-1)}{2}\left[\Phi^{\dagger} \Phi-1\right]^{2}\right\} .
\end{aligned}
$$

Note that for $\beta \geq 1$ the integral is positive definite and gives the lower bound $E \geq$ $2 \pi v^{2}$. For $\beta>1$, this bound cannot be achieved by any particular field configuration, because the last two quadratic terms give inconsistent equations for $\Phi^{\dagger} \Phi$. However, for $\beta=1$ the bound can be saturated, and the energy minimized, by solving the Bogomol'nyi equations

$$
\begin{aligned}
& D_{j} \Phi+i \epsilon_{j k} D_{k} \Phi=0, \\
& B=1-\Phi^{\dagger} \Phi .
\end{aligned}
$$

In polar coordinates, the first equation becomes $D_{\hat{r}} \Phi+i D_{\hat{\theta}} \Phi=0$. Hindmarsh applies these equations to a skyrmion ansatz

$$
\begin{aligned}
& \Phi=\left(\begin{array}{c}
f(r) e^{i \theta} \\
g(r)
\end{array}\right), \\
& \mathbf{A}=\hat{\mathbf{e}}_{\theta} \frac{a(r)}{r},
\end{aligned}
$$

with $f(0)=a(0)=0, g(\infty)=0, f(\infty)=a(\infty)=1$. Like the naive ansatz $(2.3)$, such skyrmions coincide with the semilocal string at infinity, and unwind through the vacuum manifold; however, they remain free to slip off the vacuum manifold in their cores. For them, the Bogomol'nyi equations (3.3) become

$$
\begin{aligned}
& \frac{d f}{d r}+\frac{a-1}{r} f=0, \\
& \frac{d g}{d r}+\frac{a}{r} g=0, \\
& B+\left(f^{2}-g^{2}-1\right)=0 .
\end{aligned}
$$

Note that taking $g=(\Omega / r) f$ eliminates the second equation. Hindmarsh showed that for all values $\Omega \in[0, \infty)$ there exist solutions to (3.5) satisfying the boundary 
conditions. When $\Omega=0$, the solution has $g \equiv 0$ and therefore coincides with the embedded Abrikosov-Nielsen-Olesen solution. When $\Omega$ increases, $g(r=0)$ increases and the region over which the solution's energy is spread becomes larger. For $\Omega \gg$ $1, \Phi^{\dagger} \Phi \approx 1$ everywhere, and the solution is essentially a two-dimensional texture. Therefore, for $\beta=1$ skyrmions interpolate between the semilocal string and skyrmion solutions of arbitrary size.

We now consider $\beta \neq 1$, where we no longer expect skyrmions to be static solutions. Rather they are configurations of fixed size, which tend to expand or contract. Such objects comprise a trough in configuration space: at $\beta=1$, their tension is scale-invariant and the trough is flat; however, we generically expect corrections to tilt the trough, biasing it toward either large or small skyrmions.

To understand this bias we must relate the skyrmion energy $E$ to its size $R$. By $R$ we mean the size of the region in which the gauge curvature and the covariant derivative of the scalar field differ significantly from zero. The skyrmion has scalar derivative energy of order $v^{2}$, because a covariant gradient of order $(v / R)$ extends over an area of order $R^{2}$. It has a smaller magnetic energy of order $1 / g^{2} R^{2}$. A further correction appears when we refine the approximation for the gradient energy to take into account the scalar field's freedom to move off the vacuum manifold in the skyrmion core. Suppose that inside the region of size $R, \Phi^{\dagger} \Phi \approx x^{2} v^{2}$ where $x<1$. This lowers the energy by lowering the gradient energy linearly in $\left(x^{2}-1\right)$ at a cost in potential energy quadratic in $\left(x^{2}-1\right)$. The energy then takes the form

$$
E(R, x)=\left(2 \pi \frac{v^{2} x^{2}}{R^{2}}+\frac{b}{g^{2} R^{4}}+c \lambda v^{4}\left(x^{2}-1\right)^{2}\right) \cdot R^{2}
$$

where $b$ and $c$ are undetermined coefficients of order one. Minimizing with respect to $x$ for fixed $R$ gives (to leading order in $R^{-2}$ )

$$
E(R)=2 \pi v^{2}+\left(\frac{b}{g^{2}}-\frac{\pi}{2 c \lambda}\right) \frac{1}{R^{2}}
$$

A relation between the coefficients $b$ and $c$ can be found using the special case $\beta=1$. 
The fact that the $R^{-2}$ coefficient vanishes for $\lambda=g^{2}$ allows one to simplify (3.7) to

$$
E(R)=2 \pi v^{2}+b \cdot\left(\frac{1}{g^{2}}-\frac{1}{\lambda}\right) \frac{1}{R^{2}}
$$

This order of magnitude analysis can be refined by seeking the skyrmions configurations explicitly. They are minimal energy configurations of fixed size, whose spectrum biases them toward shrinking or growing when $\beta \neq 1$. Finding them thus reduces to the question of minimizing the energy for fixed skyrmion size. There are many possible definitions for this "size" — all of which agree roughly, but not exactlywith no way to single out a particular definition as the most natural. We therefore define the size to make the calculation simple.

We define a skyrmion's "size" as its ratio of flux energy to total energy. To confine a skyrmion configuration to a smaller region, we pay a price in flux energy, as we concentrate the flux lines. For large skyrmions, this cost scales strongly, $E_{\text {mag }} \sim 1 / R^{2}$, while the total energy $E$ changes negligibly. Our parameter $\chi=E_{\text {mag }} / E$ thus acts as an "antisize."

To find a minimal energy configuration for fixed $\chi$, we introduce a Lagrange multiplier to constrain $\chi$ and minimize

$$
\bar{E}=E-\alpha\left[E_{m a g}-\chi E\right]
$$

which can be rewritten as

$$
\begin{aligned}
\bar{E} & =(1+\alpha \chi) E^{\prime} \\
& =(1+\alpha \chi) v^{2} \int d^{2} x\left\{\left|D_{i} \Phi\right|^{2}+\frac{\beta}{2}\left[\Phi^{\dagger} \Phi-1\right]^{2}+\frac{1}{2}\left(\frac{1+\alpha \chi-\alpha}{1+\alpha \chi}\right) B^{2}\right\} .
\end{aligned}
$$

In this form we see the result of constraining $\chi$ : it acts only to rescale the flux energy contribution $B^{2}$. We trade this rescaling for a rescaling of $\beta$, by rescaling 
$x \rightarrow x^{\prime}=\left(\frac{1+\alpha \chi}{1+\alpha \chi-\alpha}\right)^{1 / 2} x$, which does not affect the energy. This eliminates the factor $\left(\frac{1+\alpha \chi-\alpha}{1+\alpha \chi}\right)$ in front of $B^{2}$, and transforms $\beta$ to

$$
\beta_{e f f}=\beta\left(\frac{1+\alpha \chi-\alpha}{1+\alpha \chi}\right)
$$

This makes the problem tractable because we know how to minimize the energy when $\beta_{\text {eff }}=1$. Thus setting

$$
\alpha=\frac{\beta-1}{\beta-\chi(\beta-1)}
$$

gives solutions to the constrained problem

$$
\begin{aligned}
& f_{\beta}(r ; \Omega)=f_{1}\left(r^{\prime} ; \Omega\right) \\
& g_{\beta}(r ; \Omega)=g_{1}\left(r^{\prime} ; \Omega\right) \\
& a_{\beta}(r ; \Omega)=a_{1}\left(r^{\prime} ; \Omega\right),
\end{aligned}
$$

where $r^{\prime}=\left(\frac{1+\alpha \chi}{1+\alpha \chi}\right)^{1 / 2} x=\sqrt{\beta} r$ and $f_{1}\left(r^{\prime} ; \Omega\right), \ldots$ are the solutions for $\beta=1$. This yields a one-parameter family of skyrmions, ranging from infinite size to a smallest member at $\Omega=0$. Since this smallest member is just a rescaling of the $\beta=1$ semilocal string, it has a particle-scale size of order $(1 / \sqrt{\beta}) v^{-1}$.

Because these skyrmions have $E^{\prime}=2 \pi v^{2}$, equation (3.10) gives their spectrum exactly:

$$
E(\beta, \chi)=2 \pi v^{2} \cdot(1+\alpha \chi)=2 \pi v^{2} \cdot\left(\frac{\beta}{\beta-\chi(\beta-1)}\right)
$$

This function resolves qualitative questions about the skyrmions' flat space dynamics. $E(\chi)$ increases monotonically when $\beta>1$, and decreases monotonically when $\beta<1$. Thus skyrmions do tend to grow (to lower their flux fraction $\chi$ ) when $\beta>1$, and shrink when $\beta<1$. Moreover, no energy barriers arise in this evolution; the skyrmions evolve between one endpoint - an infinite skyrmion - and the other - a particle-scale 
skyrmion - classically and continuously. Over all these orders of magnitude, the energy (3.14)varies over at most the range

$$
E: 2 \pi v^{2} \rightarrow 2 \pi v^{2} \beta
$$

Thus the finite energy bias

$$
\Delta<2 \pi v^{2}|\beta-1|
$$

drives skyrmion expansion or contraction from cosmological to particle scales.

Note that the rescaled $\beta=1$ solutions do not solve the constrained problem for all values of $\chi$. Values of $\chi$ greater than $\beta /(1+\beta)$ are inconsistent with the Bogomol'nyi equations (3.5). For $\beta \neq 1$ the Lagrange multiplier $\alpha$ remains nonzero for all of the rescaled $\beta=1$ solutions. This means that the rescaled $\beta=1$ solutions never reach the size of the localized Abrikosov-Nielsen-Oleson solution for $\beta \neq 1$.

With these energetic tendencies thus established, we address the dynamics of cylindrically-symmetric skyrmions, described by a function $R(t)$, in flat Minkowski space. The Hamiltonian for the restricted degree of freedom $R(t)$ contains an inertia term of the form $\frac{1}{2} I v^{2} \dot{R}^{2}(t)$, corresponding to the gradient $(\partial \Phi / \partial t) \approx(v / R) \dot{R}$ spread over an area of order $R^{2} . I=O(1)$. Large skyrmions have a potential obtained by expanding the spectrum (3.14) in powers of $\chi$ :

$$
E=2 \pi v^{2}\left(1+\chi \frac{\beta-1}{\beta}+O\left(\chi^{2}\right)\right) .
$$

Given the large skyrmion relation $\chi \sim v^{-2} / R^{2}$, this agrees with the order of magnitude spectrum (3.8). The full Hamiltonian is

$$
\mathcal{H}=\frac{1}{2} I v^{2} \dot{R}^{2}+2 \pi v^{2}-\frac{b}{\lambda} \cdot(1-\beta) \frac{1}{R^{2}} .
$$

leading to the equation of motion

$$
\ddot{R}=-\frac{2 b}{\lambda I v^{2}} \cdot(1-\beta) \frac{1}{R^{3}}=\frac{-\zeta}{v^{2} R^{3}} .
$$


Thus, when $\beta<1$ skyrmions always shrink, at a rate

$$
\dot{R} \sim-\frac{1}{\sqrt{1-\beta}} \frac{1}{v R}
$$

which results in the quadratic collapse time

$$
t \sim \sqrt{\frac{1}{1-\beta}} v R^{2} .
$$

However, in the early universe, the skyrmion also experiences the pull of the universe's expansion, so that for a flat skyrmion potential

$$
\dot{R} \sim H R,
$$

where $H$ is the Hubble constant. The conflicting effects determine a cutoff size

$$
R_{c} \sim \sqrt{(H v)^{-1}},
$$

which is the geometric mean of the two length scales in the problem: the localized string core and the horizon size. For $R \gg R_{c}$, the expansion wins, and the skyrmion expands with the universe. For $R \ll R_{c}$, the skyrmion evolves instead as predicted by microphysics, collapsing slowly toward the semilocal string. Note that, in the radiation-dominated universe, the cutoff $R_{c}$ between these two regimes itself comoves with the expansion,

$$
R_{c}=\sqrt{\frac{M_{p l}}{v}} T^{-1} .
$$

This cutoff is approximate, and the large expanding skyrmions do not quite comove with the universe, due to their retarding dynamics. Thus some skyrmions which initially expand are recaptured: they fall within the comoving cutoff $R_{c}$ and succumb to dynamical collapse. However, we show below that this effect is small, leading to recapture only of skyrmions with initial sizes within an order of magnitude of $R_{c}$. Larger skyrmions expand forever, since their dynamical drag becomes ineffectual after matter-radiation equality. Before that refined analysis, however, we illustrate the relevance of the cutoff $R_{c}$ with some numbers. 
A phase transition at the GUT scale $\left(10^{16} \mathrm{GeV}\right)$ yields a semilocal string which spans $v / M_{p l}=10^{-3}$ of the initial horizon. Thus skyrmions smaller than roughly $1 / 30$ of the horizon shrink; larger ones expand. (Note that this cutoff $R_{c}$, a geometric mean, also corresponds to 30 times the semilocal core size $v^{-1}$.) To forbid initially expanding skyrmions, the correlation length must be kept smaller than $1 / 30$ of the horizon size. Folding in our promised result, that less than one order of magnitude of expanding skyrmions recollapse, this bound loosens. Skyrmions up to $1 / 3$ of the horizon size eventually collapse into semilocal string.

While this poses no restriction for GUT scale transitions, it becomes problematic as we lower $T_{c}$. A phase transition occurring 13 orders of magnitude later, at the electroweak scale $(1 \mathrm{TeV})$, yields semilocal strings which span only $v / M_{p l}=10^{-16}$ of the initial horizon. Thus skyrmions bigger than $10^{-8}$ times the horizon size (or $10^{8}$ times the semilocal core size) begin expanding. Again anticipating our promised result, only one order of magnitude of these expanding skyrmions recollapse. Thus, to prevent skyrmions which expand forever, we must require a correlation length less than $10^{-7}$ horizon sizes - a significant fine tuning of the phase transition.

We now proceed to the promised result, a careful analysis of skyrmion dynamics in an expanding FRW universe with $\Omega=1$. First, it is convenient to rewrite our equation of motion (3.19) in terms of the dimensionless co-moving size $x(t)=R(t) / a(t)$ (where $a(t)$ is the scale factor) instead of the physical size $R(t)$. One obtains

$$
\ddot{x}(t)+\frac{\zeta}{v^{2} a^{4}(t) x^{3}(t)}=0
$$

valid for the static case $\dot{a}(t)=0$. To generalize, we substitute $\ddot{x} \rightarrow \ddot{x}+3[\dot{a}(t) / a(t)] \dot{x}(t)$, to obtain the curved space equation of motion

$$
\ddot{x}(t)+\frac{3 \dot{a}(t)}{a(t)} \dot{x}(t)+\frac{\zeta}{v^{2} a^{4}(t) x^{3}(t)}=0 .
$$

For a radiation-dominated universe (with a fixed effective number of massless species), $a(t)=t_{0} \cdot\left[t / t_{0}\right]^{1 / 2}$, where $t_{0}$ is an arbitrary time scale used to fix a normalization for 
$x(t)$. Here $H(t)=1 /(2 t)$. With this choice of $a(t),(3.26)$ becomes

$$
\ddot{x}(t)+\frac{3}{2 t} \dot{x}(t)+\frac{\zeta}{v^{2} t_{0}^{2}}\left(\frac{t_{0}}{t}\right)^{2} \frac{1}{x^{3}(t)}=0 .
$$

Rewritten in terms of logarithmic time $s=\ln \left[t / t_{0}\right],(3.27)$ becomes

$$
\frac{d^{2} x}{d s^{2}}+\frac{1}{2} \frac{d x}{d s}+\frac{\zeta}{\left(v t_{0}\right)^{2}} \cdot \frac{1}{x^{3}(s)}=0 .
$$

At this point, we normalize $x(t)$ so that at the phase transition $t=t_{p t}, x=$ 1 corresponds to the physical size of the Abrikosov-Nielsen-Olesen vortex - that is, $a\left(t_{p t}\right) \approx v^{-1}$. This occurs for $t_{0}=M_{p l}^{-1}$, so that (3.28) becomes

$$
\frac{d^{2} x}{d s^{2}}+\frac{1}{2} \frac{d x}{d s}+\zeta\left(\frac{M_{p l}}{v}\right)^{2} \frac{1}{x^{3}(s)}=0 .
$$

Note this equation is invariant to translation in logarithmic time $s$; we thus set $s(t=$ $\left.t_{p t}\right)=0$. We want to know at what later value of $s$ a skyrmion initially at rest with size $x$ completes its collapse. Since the final stages of collapse occur rapidly, it is justified to assume that the collapse completes when $x=0$.

Note that equation (3.29) describes dynamics in the "time" $s$, where the force acts always to push the velocity and acceleration (both initially zero) in the same direction. Thus a positive acceleration always reduces the velocity from its terminal value,

$$
\frac{1}{2} \frac{d x}{d s}+\zeta \cdot\left(\frac{M_{p l}}{v}\right)^{2} \cdot \frac{1}{x^{3}(s)}=0 .
$$

Using this terminal velocity, then, we underestimate the collapse time. We obtain

$$
s_{c o l}(x)=\int_{x}^{0}\left(\frac{d x}{d s}\right)^{-1} d x \gtrsim \frac{1}{8 \zeta} \cdot\left(\frac{v}{M_{p l}}\right)^{2} x^{4} .
$$

Recall that $t_{c o l}=t_{p t} \cdot e^{s}$, so rather modest values of $s$ can represent exceedingly long times. We see this by calculating the size of the smallest skyrmions which fail 
to collapse before recombination $\left(T_{\text {rec }} \approx 1 \mathrm{eV}\right)$. Such skyrmions have collapse times larger than $s_{\max }=\ln \left[t_{p t} / t_{r e c}\right] \approx \ln \left[v^{2} / T_{r e c}^{2}\right]$. This bounds their size

$$
x \gtrsim x_{c} \approx\left(\frac{M_{p l}}{v}\right)^{1 / 2} \cdot\left(8 \zeta s_{\max }\right)^{1 / 4} .
$$

We compare this with our first order of magnitude analysis. Equation (3.24) gives the naive physical cutoff $R_{c} \sim \sqrt{M_{p} l / v} v^{-1}$ at the phase transition. From our normalization $a\left(t_{p t}\right)=v^{-1}$. Thus this more careful analysis modifies the naive cutoff as follows:

$$
x_{c} \approx x_{c, \text { naive }} \cdot\left(8 \zeta s_{\max }\right)^{1 / 4} \approx x_{c, \text { naive }} \cdot 2\left(\ln v / T_{\text {rec }}\right)^{1 / 4}
$$

The correcting factor accounts for the fact that initially expanding skyrmions eventually recollapse, over the cosmological time scales we consider here. Given the length of those time scales, the correction is amazingly weak. For a GUT scale phase transition, $v / T_{\text {rec }}=10^{25}$ gives only $x_{c} \sim 6 x_{c, \text { naive. }}$. That is, only skyrmions 6 times bigger than those which begin collapsing instantly are ever recaptured, even as we evolve through 25 orders of magnitude in temperature. For an electroweak phase transition, things change little: $v / T_{\text {rec }}=10^{12}$ gives $x_{c} \sim 5 x_{c, \text { naive }}$.

For a matter-dominated universe, the conditions for collapse become even less favorable, because the expansion of the universe slows less rapidly. In a matterdominated universe, $a(t)=t_{0} \cdot\left[t / t_{0}\right]^{2 / 3}$, so $(3.27)$ becomes

$$
\ddot{x}(t)+\frac{2}{t} \dot{x}(t)+\frac{\zeta}{v^{2} t_{0}^{4}} \cdot\left(\frac{t_{0}}{t}\right)^{8 / 3} \cdot \frac{1}{x^{3}(t)}=0 .
$$

Again using logarithmic time $s=\ln \left[t / t_{0}\right]$, one obtains

$$
\frac{d^{2} x}{d s^{2}}+\frac{1}{2} \frac{d x}{d s}+\frac{\zeta}{\left(v t_{0}\right)^{2}} \cdot e^{-(2 / 3) s} \frac{1}{x^{3}(s)}=0 .
$$

The crucial difference between (3.28) [radiation domination with $p \approx \rho$ ] and (3.35) [matter domination with $p \ll \rho$ ] is the presence of the factor $e^{-(2 / 3) s}$, which 
causes the potential term in the matter-dominated universe to become feeble exponentially, so that large skyrmions never collapse, even in the $t \rightarrow \infty$ limit. Therefore, one expects most skyrmions that survive till matter domination to survive forever without collapsing.

\section{Completely-Gauged Nontopological Strings}

As mentioned in the Introduction, the stability of embedded strings does not rely on the existence of global symmetries. Instead, the global symmetries can be weakly gauged (by introducing new gauge fields whose couplings are much smaller than the pre-existing ones), without destroying the stability of semilocal solutions. However, making the gauge couplings roughly comparable does destroy their stability. Vachaspati and collaborators studied the model (2.1) with an $S U(2)$ gauge field added (i.e., the Standard Electroweak Model) and found embedded $Z$-strings which are stable for values of $\sin \theta_{W}$ close to one ${ }^{[8,11,12]}$. These strings carry mostly $U(1)$ flux, with a small admixture of $S U(2)$ flux, which is energetically disfavored because $g^{\prime} \gg g$. (Here $g$ is the $S U(2)$ gauge coupling and $g^{\prime}$ the $U(1)$ gauge coupling.) To deform such an electroweak vortex to a configuration that is pure gauge, one must first turn the $U(1)$ flux into energetically more costly $S U(2)$ flux, which involves climbing over an energy barrier of order $1 /\left(g^{2} r_{v o r}^{2}\right)$.

The most important consequence of gauging the global symmetry is the absence of large skyrmions. Large skyrmions arise only in embedded models that are "partially gauged" - that is, models which have directions on the vacuum manifold that cannot be compensated by gauge fields. In this case, unwinding the gauge orbit at spatial infinity - by contracting it in the vacuum manifold - always has some gradient energy cost. To first order, that cost is scale-invariant and of order $v^{2}$. It is borne most efficiently by the skyrmions, or textured string configurations.

In models that are "completely gauged," (such as the electroweak model with $g \neq 0$ ), every direction on the vacuum manifold can be compensated by gauge fields. Thus, when we contract the gauge orbit in the vacuum manifold, we can offset all 
gradients by exciting the weakly coupled gauge field. This carries a cost in flux energy of order $1 / g^{2} R^{2}$ - expensive compared to $U(1)$ flux, but negligible compared to the finite gradient energy of skyrmions. Thus we see the key difference between semilocal and completely gauged theories: in semilocal theories, even infinitely delocalized configurations with $U(1)$ winding carry an energy of order $v^{2}$; whereas, in completely gauged theories, configurations with such delocalized winding can cost negligible energy.

Note that these delocalized configurations, in the completely gauged case, invariably expand, driven by their flux energy. Thus completely gauged embedded strings get only one opportunity in the competition to resolve Kibble frustration. They must form at the phase transition, or not at all, as their negligible-energy competitors expand forever once they form.

The absence of large skyrmions also implies that completely-gauged nontopological strings can break, ${ }^{[21]}$ without forming a fat skyrmionic string of comparable or often larger energy to fill the gap. As Nambu explained, this does not mean that electroweak strings can end into nothingness ${ }^{[7]}$. The string carries a $U(1)_{Y}$ magnetic hypercharge and $U(1)$ lines of magnetic induction must close on themselves. This means that an "electroweak" string ends on something resembling a magnetic monopole. Inside the string there is $Z$-magnetic flux, which is a mixture of $S U(2)$ magnetic flux and $U(1)_{Y}$ magnetic flux. Where the string ends, this $Z$-magnetic flux turns into $Q$-magnetic flux in such a way that $Y$-magnetic flux is conserved. The end of the string is not really a magnetic monopole because the Dirac string is physical and the energy density at the end of the string is comparable to the energy density elsewhere in the core of the string. Therefore, in order not to collapse, a finite segment of electroweak string must rotate so that the velocity at the end of the string is approximately the velocity of light.

There are also models with completely-gauged nontopological strings that can end into nothingness. Vachaspati and Barriola ${ }^{[17]}$ found a "W"-string solution that can be embedded in the Standard model. In this solution the $U(1)_{Y}$ gauge field is 
not excited; therefore, the string is free to break without any magnetic flux filling the gap.

\section{Semilocal Strings and Cosmology}

We now discuss the formation and evolution of a network of nontopological string in the early universe. We conclude that the usual analysis of string network formation, valid for topological cosmic strings, cannot naively be extended to nontopological strings. These are both more difficult to form and more fragile after formation than their topological counterparts.

For topological strings, the standard picture for formation is the following. ${ }^{[22,23,24]}$ As the universe cools, a phase transition takes place and the Higgs field $\Phi(x)$ acquires a nontrivial vacuum expectation value, with the orientation of $\Phi(x)$ uncorrelated over length scales larger than the correlation length $\xi$. This correlation length $\xi$ corresponds for second-order phase transitions to the length scale where critical slowing down prevents $\xi$ from further growth and for first-order phase transitions to the separation between bubbles. In either case, causality bounds $\xi$ to be smaller than the Hubble length $H^{-1}$.

Typically, to simulate string formation in this process, one takes a lattice with spacing $\xi$ and lays down random uncorrelated orientations of the Higgs field at the sites of the lattice ${ }^{[25]}$. A rule is established for interpolating $\Phi(x)$ along the links connecting adjacent sites, so that the winding number $N$ throug h each plaquette can be calculated. For example, for the $U(1)$ abelian Higgs model, a three-dimensional triangular lattice can be used, with $\Phi(x)$ randomly set to $1, e^{i 2 \pi / 3}, e^{i 4 \pi / 3}$ (which we shall denote as $1,2,3$, respectively) at each site. 123 around a plaquette (and its two cyclic permutations) corresponds to a wind ing number $N=+1,213$ (and its cyclic permutations) corresponds to $N=-1$, and the other 21 possibilities correspond to $N=0$.

For topological strings, the winding number $N$ through each plaquette is equal to the number of strings minus the number of antistrings passing through the plaquette. 
For the $U(1)$ example each tetrahedral simplex has either no strings passing through it, or one string entering and leaving the simplex. Therefore, there is no ambiguity in determining the trajectories of the strings from the winding numbers on the plaquettes. The end result is a scale-invariant distribution of closed loops (dominated by small loops) and some open infinite strings. ${ }^{[25,1]}$

Unlike theories with topological strings, "semilocal" systems can accommodate winding boundary conditions through a multiplicity of low-energy configurations. They can untwist internally by producing a tightly localized semilocal core, or a skyrmionic core with size up to the correlation length. Thus, for nontopological strings, the winding number $N$ does not determine the number of "strings" passing through a plaquette. This requires more information than simply the behavior of the fields on the boundary of the surface. When $\xi$ is much larger than $v^{-1}$ (the thickness of the semilocal string), there is a high probability that $N \neq 0$ will correspond to a large delocalized skyrmion, and not to a localized string.

The distinction between "localized" and "delocalized" is not a sharp one. On the one hand, if frustration resolves on scales larger than $v^{-1}$ but at the same time smaller than the length scale $L_{f}$ characterizing the frustration density (so that voids may appear between the fattened skyrmionic strings), the result is best described as a network of fattened string. But, on the other hand, if skyrmions have a thickness comparable to $L_{f}$, then perturbations in energy density — at least initially — will hardly resemble that of a string network. Because there are no voids (where the energy density perturbation is negligible), the skyrmions will more closely resemble a global texture. While it is reasonable in former case to count skyrmions as "strings," in the latter case to do so would be misleading.

For topological strings, the lattice model - as crude as it is - guarantees that there are at least some open infinite strings. As demonstrated by simulations and analytical models, an initial string distribution with some open infinite strings rapidly settles down to what is known as the "scaling solution" ${ }^{[24,26]}$, in which there is approximately one infinite string per horizon volume and a scale-invariant distribution of large string 
loops. The expansion of the universe tends to stretch and straighten out long cosmic strings. If cosmic strings did not intercommute, but instead merely passed through each other upon colliding, the number of strings per horizon volume would rapidly grow, and cosmic strings would eventually dominate the mass density of the universe. But instead abelian cosmic strings always intercommute upon colliding. ${ }^{[27,28,29]}$ Intercommuting reduces the amount of infinite string per unit co-moving volume by allowing loops to be chopped off and by preventing infinite strings from becoming straightened out and stretched. Causality prevents the density of infinite strings from dropping below approximately one infinite string per horizon volume. The scaling solution is stable in the sense that any initial distribution of cosmic strings that contains some infinite strings will approach the scaling solution as $t \rightarrow \infty$.

The essential input required for a distribution of cosmic strings to approach the scaling solution is that there initially be a nonvanishing density of infinite strings. There is no compelling reason to believe that any open, infinite semilocal strings are generically produced at the phase transition. If one assumes that a frustrated plaquette has some probability $p<1$ of corresponding to a semilocal string and a probability $(1-p)$ of corresponding to a large skyrmion, then one will obtain closed loops of semilocal string and open segments of semilocal string that connect to skyrmions on both ends. The distribution of closed loops initially will be exponentially suppressed from a $d n / d R \sim R^{-4}$ scale-invariant distribution, and there will be no infinite, open semilocal strings - at least immediately after the phase transition.

Subsequently, for $\beta<1$, finite segments of semilocal string lengthen, and some of the skyrmionic string starts to collapse. But these two mechanisms cannot produce infinite semilocal strings unless the correlation length is restricted to small values, less than approximately $10 \cdot\left(M_{p l} / v\right)^{1 / 2} v^{-1}$. Larger correlation lengths give rise to delocalized skyrmionic string, which spreads with the Hubble flow and expands forever. Finite-length segments of semilocal string whose flux flows into such large, forever expanding regions of delocalized flux effectively stop their growth, because their small energetic advantage must act against a huge and increasing inertia. Even smaller skyrmions, which begin to collapse, offer no guarantee of eventual semilocal string. 
Their delocalized flux and frustration can also relax by interacting and subsequently annihilating with nearby delocalized flux and frustration. In other words, skyrmions can disappear without ever becoming localized.

We have yet to consider thermal effects, which destabilize the semilocal string for $\beta$ close to one. Immediately after the phase transition, the temperature is of order $v$, but as $\beta \rightarrow 1$ from below the energy gap between semilocal strings and delocalized skyrmions becomes negligible compared to $v$. Thus one expects thermal bombardment to destabilize any short segments of semilocal string formed near the phase transition.

For "completely-gauged" nontopological strings, the prospects for formation are even more bleak because there are no competing large skyrmion configurations, with energy per unit length of order $v^{2}$. Completely-gauged nontopological strings that do not carry any abelian magnetic flux can simply end into pure vacuum. This fact implies that even if such strings are classically stable (that is, stable against small perturbations), they break at a finite rate due to quantum tunneling processes. The Euclidean action for the instanton for this process is not very large because there is no need to create heavy monopoles. ${ }^{[2]}$. Thermal fluctuations also break completelygauged nontopological strings. When completely-gauged nontopological strings carry some abelian magnetic flux, the situation differs in one minor respect. The string cannot simply end because $\nabla \cdot \mathbf{B}=0$ for an abelian magnetic field. This means that where the string breaks, there must be magnetic flux in the gap connecting the two string segments. However, the energy between the two strings is minuscule because there is no scalar gradient energy. Therefore, energy per unit length in a gap of width $R$ is roughly $1 /\left(g^{\prime} R\right)^{2}$.

Completely-gauged nontopological strings are unlikely to form, except perhaps for some small loops, because the competing large configurations have completely negligible energy. Instead of competing with skyrmions of comparable tension, biased to collapse into localized string, they compete with delocalized configurations of negligible tension which tend to expand. After the phase transition there are no de- 
localized strings lurking around with the potential of collapsing into localized strings. Localized strings either form immediately after the phase transition or not at all.

\section{Concluding Remarks}

Nontopological embedded string solutions are more fragile than their topologically stable counterparts. A nontopological string can be unwound by supplying a finite energy per unit length. If the nontopological string is "semilocal," the string unwinds by turning into a fat "skyrmionic" string whose radius increases at an asymptotically constant velocity. If the nontopological string is "completely gauged," it can disappear, merely turning into outgoing particles and radiation. By contrast, it requires an energy proportional to $R$ to delocalize the winding of a topologically stable string to a cylinder of radius $R$. Therefore, an infinite amount of energy per unit length is required to unwind a topologically-stable string.

In a cosmological context, the presence of stable nontopological strings in a model does not mean that a string network, described by the scaling solution, invariably forms due to the Kibble mechanism. Formation of such a network can occur only for

correlation lengths $\xi \lesssim 10 \cdot \sqrt{M_{p l} / v} \cdot v^{-1}$ for semilocal strings, and $\xi \lesssim(1 / g) v^{-1}$ for completely-gauged strings. Furthermore, even if a completely-gauged string network forms initially, it is later destroyed by thermal and instanton effects.

However, even if a string network does not form in a semilocal model owing to the role of expanding delocalized "skyrmions," this does not mean that the resulting skyrmions are cosmologically uninteresting. Density perturbations will still be produced and could be relevant to structure formation; however, the density perturbations will not be "stringy" - that is, they will not have a filament-like structure.

In this paper we have studied the dynamics of delocalized skyrmion configurations, both in a static universe and in an spatially flat expanding universe. We found that for large skyrmions the potential scales as $E(R) \sim(\beta-1) R^{-2}$, becoming exceedingly weak for large $R$. Therefore, the expansion of the universe becomes relevant-and 
even dominant - for $R$ substantially smaller than the horizon size. Skyrmions of a rather modest size are swept away by the Hubble flow and never collapse, despite the fact that their collapse would lead to lower energy. We consider the relevance of these competing fat skyrmionic strings to cosmology, demonstrating that they prevent the extension of the standard picture for string formation, valid for topologically stable strings, to semilocal strings. One of the disappointing results of this investigation is the fact that the formation and evolution of "nontopological" strings depend crucially on the length scales which arise at the phase transition. By contrast, for "topological" strings (and other types of topologically-stable defects), the details of the phase transition become largely irrelevant at later times. For topologically-stable strings, the fact that the details of the phase transition are quickly erased leads to more generic, model independent predictions.

\section{Acknowledgments}

We would like to acknowledge helpful discussions with Andrew Liddle, John Preskill, Tanmay Vachaspati, Alexander Vilenkin, David Weinberg, and Frank Wilczek.

\section{REFERENCES}

1. J. Preskill, "Vortices and Monopoles," in Architecture of the Fundamental Interactions at Short Distances, ed. P. Ramond and R. Stora (North-Holland, Amsterdam, 1987).

2. A. Vilenkin, "Cosmic Strings and Domain Walls," Phys. Rep. 121, 263 (1984)

3. G. Gibbons, S. Hawking and T. Vachaspati, Eds., The Formation and Evolution of Cosmic Strings, Cambridge: Cambridge U. Press (1990).

4. A. Abrikosov, "On the Magnetic Properties of Superconductors of the Second Group," Soviet Phys. JETP 5, 1174 (1957).

5. H. Nielsen and P. Oleson, "Vortex-Line Models for Dual Systems," B61, 45 (1973). 
6. T. Vachaspati and A. Achúcarro, "Semilocal Cosmic Strings," Phys. Rev. Lett. 44, 3067 (1991).

7. Y. Nambu, "Stringlike Configurations in the Weinberg-Salam Theory," Nucl. Phys. B130, 505 (1978).

8. T. Vachaspati, "Vortex Solutions in the Weinberg-Salam Model," Phys. Rev. Lett. 68, 1977 (1992).

9. M. Hindmarsh, "Existence and Stability of Semilocal Strings," Phys. Rev. Lett. 68, 1263 (1992).

10. A. Achúcarro, K. Kuijken, L. Perivolaropoulos and T. Vachaspati, "Dynamical Simulations of Semilocal Strings," Harvard-Smithsonian CfA Preprint 3384 (1992).

11. M. James, L. Perivolaropoulos and T. Vachaspati, "Detailed Stability Analysis of Electroweak Strings," Tufts Preprint 1992

12. L. Perivolaropoulos and T. Vachaspati, "On the Stability of Electroweak Strings, Harvard-Smithsonian CfA Preprint 3388 (1992).

13. G. Gibbons, M. Ortiz, F. Ruiz and T. Samols, "Semilocal Strings and Monopoles," DAMTP HEP 92-09 (1992).

14. R. Leese and T. Samols, "Interactions of Semilocal Vortices," DAMTP 92/40 (1992).

15. T. Vachaspati and R. Watkins, "Bound States Can Stabilize Electroweak Strings," TUPT-92-10 (1992).

16. R. Holman, S. Hsu, T. Vachaspati and R. Watkins, "Metastable Cosmic Strings in Realistic Models," Phys. Rev. D46, 5352 (1992).

17. T. Vachaspati and M. Barriola, "A New Class of Defects," Phys. Rev. Lett. 69, 1867 (1992).

18. J. Preskill, "Semilocal Defects," CALT-68-1787 (1992).

19. M. Hindmarsh, "Semilocal Toplogical Defects," DAMTP-HEP-92-24 (1992). 
20. E.B. Bogomol'nyi, "Stability of Classical Solutions," Sov. J. Nucl. Phys. 24, 449 (1976).

21. J. Preskill and A. Vilenkin, "Decay of Metastable Topological Defects," Harvard Preprint HUTP-92-A018 (1992).

22. T.W.B. Kibble, "Topology of Cosmic Domains and Strings," J. Phys. A9, 1387 (1976).

23. Y. Zel'dovich, "Cosmological Fluctuations Produced Near a Singularity," Mon. Not. Astr. Soc. 192, 663 (1980).

24. A. Vilenkin, "Cosmological Density Fluctuations Produced by Vacuum Strings," Phys. Rev. Lett. 46, 1169 (1981), Erratum: 46, 1496 (1981).

25. T. Vachaspati and A. Vilenkin, "Formation and Evolution of Cosmic Strings," Phys. Rev. D30, 2036 (1984).

26. T.W.B. Kibble, "Evolution of a System of Cosmic Strings," Nucl. Phys. B252, 227 (1985).

27. E.P.S. Shellard, "Cosmic String Interactions," Nucl. Phys. B283, 624 (1987)

28. R. Matzner, Comput. Phys. 2, 51 (1988).

29. K. Moriarty, E. Meyers and C. Rebbi, "Interactions of Cosmic Strings," in F. Acceta and L. Krauss, Cosmic Strings: The Current Status, Singapore: World Scientific (1988). 